Version in english:

http://www.conhecer.org.br/enciclop/2020C/avaliacao\%20da\%20fauna2.pdf
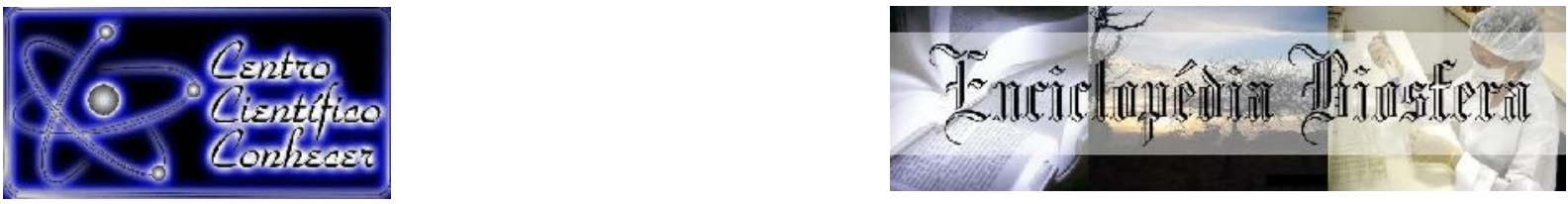

\title{
AVALIAÇÃO DA FAÚNA EDÁFICA E DESENVOLVIMENTO DO CACAU INOCULADO COM FUNGOS MICORRÍZICOS
}

Aila da Silva Mendes ${ }^{1}$, Anastacia Pavão Oliveira ${ }^{2}$, Alini Oliveira dos Santos ${ }^{2}$ Andréa Hentz de Mello ${ }^{3}$

Engenheira Agrônoma - Universidade Federal do Sul e Sudeste do Pará (Unifesspa), Marabá-PA, Brasil. E-mail: aillamendes@hotmail.com

${ }^{2}$ Mestrandas em Dinâmicas Territoriais e Sociedade na Amazônia - Universidade Federal do Sul e Sudeste do Pará (Unifesspa), Marabá-PA, Brasil.

${ }^{3}$ Professora do Mestrado em Dinâmicas Territoriais e Sociedade na Amazônia, (Unifesspa), Marabá-PA, Brasil

Recebido em: 15/08/2020 - Aprovado em: 15/09/2020 - Publicado em: 30/09/2020 DOI: 10.18677/EnciBio_2020C10

\begin{abstract}
RESUMO
Os solos da região Amazônica são representados em sua maioria por Latossolos e Argissolos de alta acidez e baixa fertilidade. Nesse sentido busca-se nesta região alternativas de manejo eficientes na utilização dos recursos naturais. Este trabalho, teve o objetivo de avaliar a qualidade biológica do solo e o desenvolvimento do cacau inoculado com fungos micorrízicos a campo, bem como parâmetros de crescimento do cacau. Foram coletadas amostras em cada área de plantio do cacau para identificação dos organismos edáficos do solo. Em seguida, foram plantadas as mudas de cacau inoculadas e não inoculadas com Fungos Micorrízicos Arbusculares (FMAs) do gênero Glomus. Aos 19 meses após o plantio as mudas foram avaliadas quanto a taxa de sobrevivência, diâmetro do colo, altura da planta e número de folhas. Os organismos identificados foram as aranhas, nematóides e colêmbolos na área não inoculada e na área de cacau inoculado foi encontrada maior diversidade de organismos, como: nematóides, acaros, FMAs, ascosporos e colembolos. As mudas de cacau inoculadas tiverem melhor desempenho em todos os parâmetros avaliados. A introdução de insumos biológicos (FMAs) nas áreas de cacau contribuiu para a melhoria da qualidade biológica do solo e adaptação das mudas em condições de estresse.
\end{abstract}

PALAVRAS-CHAVE: Biologia do Solo; Cacauicultura; Tecnologias de produção.

\section{EVALUATION OF THE EDAPHIC FAUNA AND DEVELOPMENT OF COCOA INOCULATED WITH MYCORRHIZAL FUNGI}

\begin{abstract}
The soils of the Amazon region are represented mostly by Oxisols and Argisols with high acidity and low fertility. In this sense, this region seeks alternatives for efficient management in the use of natural resources. This work aimed to evaluate the biological
\end{abstract}


quality of the soil and the development of cocoa inoculated with mycorrhizal fungi in the field, as well as parameters of cocoa growth. Samples were collected in each cocoa plantation area to identify soil edaphic organisms. Then, inoculated and non-inoculated cocoa seedlings with Glomus FMAs were planted. At 19 months after planting, the seedlings were evaluated for survival rate, stem diameter, plant height and number of leaves. The identified organisms were spiders, nematodes and collemboli in the noninoculated area and in the inoculated cocoa area, a greater diversity of organisms was found, such as: nematodes, mites, Fmas, aspaspores and colembolos. The inoculated cocoa seedlings perform better in all evaluated parameters. The introduction of biological inputs (FMAs) in the cocoa areas contributed to improving the biological quality of the soil and adapting the seedlings under stress conditions.

KEYWORDS: Cocoa culture; Soil Biology; Production technologies.

\section{INTRODUÇÃO}

O cultivo do cacaueiro tem ganhado cada vez mais destaque na região Amazônica, principalmente no estado do Pará que é atualmente o maior produtor do cacau no Brasil (IBGE 2017), surgindo como uma nova perspectiva nos modelos de cultivo em diversos locais da Amazônia, ao ser incorporado como um dos componentes principais de sistemas Agroflorestais. A cultura tem diminuído os impactos da agricultura migratória e contribuído na diversificação dos cultivos pois incorpora aspectos ambientais e econômicos ao sistema de produção (SANTOS; SILVA, 2017) e se adapta a sistemas mais diversificados, compostos por diferentes espécies florestais. A expansão do cultivo do Theobroma cacao no Pará, tem sido incentivado por programas do governo do Estado com medidas para alavancar, qualificar mudanças na base técnica, econômica, social e ambiental da cadeia produtiva do cacau (OLIVEIRA, 2016), sendo assim, consideradas estratégias para o desenvolvimento sustentável da produção (SEDAP, 2016).

Mantovanelli et al. (2016) afirmam que a região Amazônica normalmente apresenta solos pobres com baixa retenção de nutrientes devido à baixa concentração de material orgânico incorporado no solo como ocorrência de campos naturais que apresentam alta concentração de alumínio, resultando em baixos valores e pH. Sabendo que o desenvolvimento satisfatório do cacaueiro requer solos com fertilidade adequada, profundos e bem drenados, cultivos nessas condições podem levar as plantas a apresentar deficiência nutricional, resultando em restrições no crescimento das plantas e na diminuição da sustentabilidade da produção (RODRIGUES et al., 2018).

Isso pode ser agravado se os produtores estabelecerem seus cultivos sem se preocupar com fatores relacionados à conservação do solo, distribuição espacial, sombreamento, e qualidade da semente, implicando assim em problemas relacionados à instabilidade do cultivo e produtividade do cacau (JESUS et al., 2013).

Diante disso, busca-se a inserção de alternativas sustentáveis no manejo deste cultivo, principalmente aquelas que dizem respeito à melhoria da qualidade do solo. Realizar o manejo sustentável deste é extremamente importante, pois o solo é tido como um recurso vivo e dinâmico. A partir destas condicionantes o estudo da qualidade do solo parte da sua capacidade de ser funcional, respeitando os limites estabelecidos pelo ecossistema e as formas de uso da terra, de modo a preservar e sustentar a produção biológica e a qualidade ambiental, contribuindo desta forma para saúde dos 
seres vivos e integridade dos ecossistemas (SCHEMBERGUE et al., 2017; BALOTA, 2018).

A meso e macrofauna do solo desempenha funções fundamentais para a manutenção do ecossistema, sendo considerada bioindicadora de qualidade do solo, visto que as ações antrópicas, acarretam redução da diversidade e abundância de organismos da mesofauna. O estudo desse grupo é relevante para o conhecimento do estado de degradação do solo, sugere-se que mais estudos sejam feitos sobre a diversidade da mesofauna do solo (BERUDE et al., 2015).

Fungos micorrízicos arbusculares constituem parte significativa da biomassa microbiana do solo e estão envolvidos diretamente em processos essenciais da interface solo-planta. A inoculação com os fungos proporcionam a maior conteúdo de $\mathrm{K}$ e $S$, além do maior crescimento da parte aérea e das raízes das plantas, proporcionando também maior eficiência de utilização de nutrientes, principalmente de $P$, influenciando positivamente no seu crescimento e tornando-a mais resistente a fatores bióticos e abióticos (RODRIGUES et al., 2018; NASCIMENTO et al., 2018).

Diante destes fatores que primam à qualidade do solo como condicionante para o bom desenvolvimento do cacaueiro, os FMAs se apresentam como uma alternativa viável em beneficio desta cultura, pois segundo Gomide et al. (2014), estes fungos são de primordial importância na manutenção e dinâmica dos ecossistemas, beneficiando diretamente as regiões tropicais, onde a reserva mineral de nutrientes no solo é escassa, resultando na carência de uma estrutura biológica funcional nestas regiões. Sendo necessário conhecimento destes organismos, pois são fundamentais para melhoria da produtividade agrícola, uma vez que contribuem beneficamente para sustentabilidade dos agroecossistemas (SIQUEIRA et al., 2002).

Nota-se um avanço nos estudos com a inoculação e dependência simbiótica de fungos micorrizicos em algumas frutíferas e sabe-se que o uso de espécies de FMA proporciona melhora no estado nutricional na produção de mudas cultivares utilizadas e maior desenvolvimento vegetativo da planta (FARIAS et al., 2014: GOMES et al., 2018). No entanto, poucos estudos avaliam a interação e a dependência micorrízica entre fungo e a cultura do cacau. Berude et al., (2015) sugerem a realização de novos estudos sobre as aplicações micorrizas e o desenvolvimento das plantas inoculadas.

Sendo a cultura do cacau de grande importância na região amazônica e a crescente busca por alternativas sustentáveis para a produção agrícola, neste trabalho objetivou-se avaliar a fauna edáfica do solo e os parâmetros de crescimento do cacau, após a inoculação com os fungos micorrízicos no PA Palmares II em Parauapebas - PA.

\section{MATERIAL E MÉTODOS}

A área experimental correspondeu a um monocultivo de cacau de 1 ha, localizado no lote 32 do PA Palmares II em Parauapebas - PA. Os solos do lote são caracterizados como Latossolos Vermelho Amarelo distróficos, sendo que a cobertura vegetal do assentamento é composta por mata, pastagem, capoeira e roça.

O preparo do solo para o plantio do cacau ocorreu através do sistema de corte e queima, onde as 422 mudas foram plantadas e inoculadas com fungos micorrízicos arbusculares e 422 mudas não inoculadas com fungos micorrízicos arbusculares, dispostas em 6 linhas, sendo que o espaçamento entre linhas foi de $1,5 \times 1,5 \mathrm{~m}$ e o espaçamento entre plantas de 3,0 x 3,0 m e a área total foi dividida em 0,5 ha inoculado e 0,5 ha não inoculado. O delineamento utilizado foi o inteiramente casualizado, 
seguindo as recomendações de Pimentel - Gomes (1987).

As plantas foram inoculadas no momento do plantio, com uma mistura de areia e inóculo de FMAs da espécie Glomus clarum provenientes do banco de inóculo da Faculdade de Ciências Agrárias de Marabá, sendo que em cada cova de plantio foram colocadas aproximadamente $3 \mathrm{~g}$ do inóculo.

A avaliação das mudas no campo foi realizada dezenove meses após o plantio em campo, sendo que as avaliações ocorreram no formato de mutirão agroecológico, onde contou-se com a colaboração de alunos de graduação do curso de Agronomia 2011, onde foram avaliados os parâmetros de crescimento e desenvolvimento, como taxa de sobrevivência, diâmetro do colo, altura da planta e número de folhas. Os dados foram analisados com o auxílio do software SISVAR 5.1 Build 72, de acordo com as recomendações de Pimentel - Gomes (1987). Foi realizada análise de variância e o teste Tukey para a comparação de médias $(P<0,05)$.

Na figura 1 observa-se a realização da avaliação dos parâmetros de crescimento e diâmetro do colo do cacau estabelecido na área.

FIGURA 1- Avaliação dos parâmetros de crescimento e diâmetro do colo do cacau no PA Palmares, Parauapebas - PA.
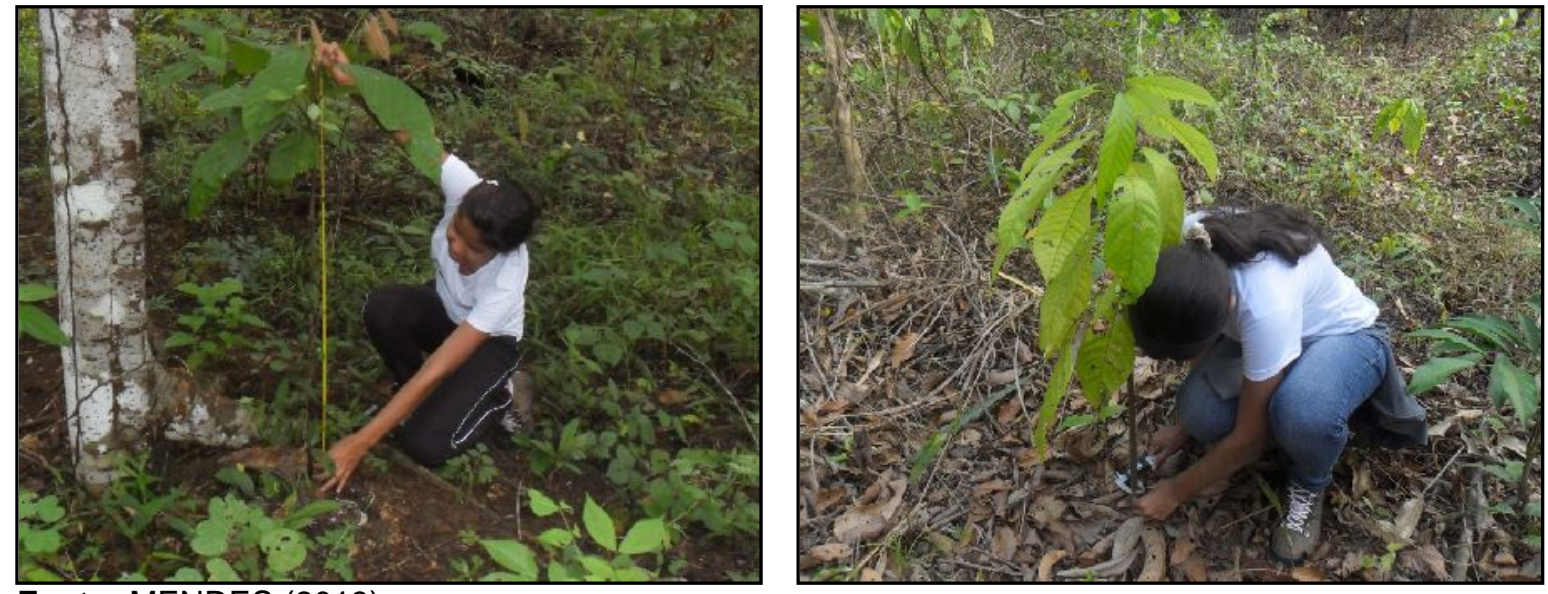

Fonte: MENDES (2013).

Foram coletadas 10 amostra simples de solo na área de monocultivo do cacau inoculado e 10 amostras simples de solo na área de monocultivo do cacau não inoculado, sendo que cada amostra simples corresponde a um volume de solo coletado de um ponto aleatório da gleba, a uma profundidade de $10 \mathrm{~cm}$ em uma área de $100 \mathrm{~m}^{2}$, segundo a metodologia de Lemos (2000) (Figura 2). As amostras de solo foram homogeneizadas, constituindo amostras compostas, sendo separadas aproximadamente 100 gramas de solo de cada área em sacos plásticos que foram encaminhadas para o Laboratório de Microbiologia da Faculdade de Ciências Agrárias de Marabá, onde foram mantidas a temperatura ambiente para a extração dos organismos do solo e posterior caracterização e identificação das espécies de fungos micorrízicos. 
FIGURA 2- Realização da coleta de amostras do solo na área de plantio do cacau inoculado e não inoculado com fungos micorrízicos no PA Palmares, Parauapebas - PA.

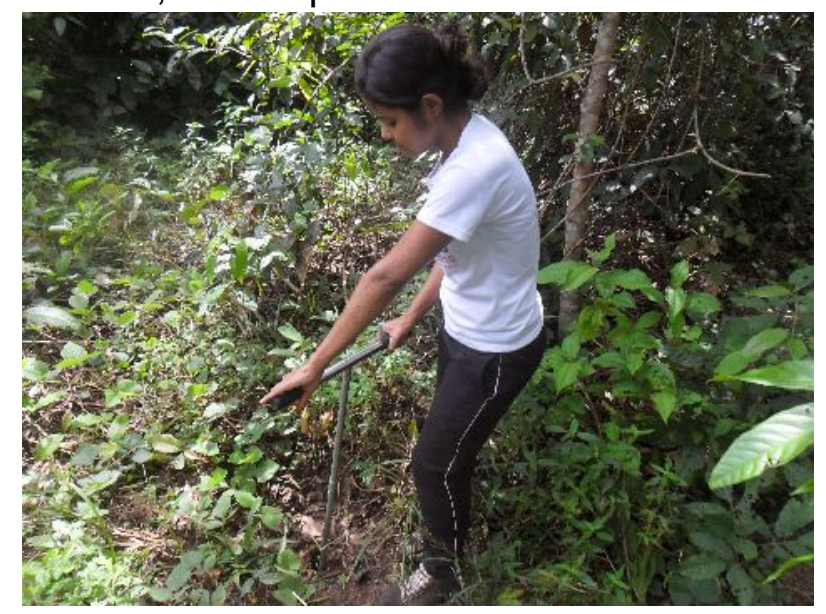

Fonte: MENDES (2013)

A técnica empregada para avaliação dos organismos presentes nas amostras de solo foi a de peneiramento úmido de Gerdeman e Nicolson, (1963) e centrifugação em água e sacarose a 40\% (JENKINS, 1964). A identificação dos gêneros e espécies encontradas foram feitas através da observação das características morfológicas externas de sua formação com o auxílio de uma lupa esteroscópica. Em seguida, foram feitas lâminas microscópicas para posterior identificação e classificação.

\section{Qualidade Biológica do Solo}

\section{RESULTADOS E DISCUSSÃO}

A partir da análise biológica realizada nas amostras de solo da área de monocultivo de cacau, identificou-se a diversidade de organismos indicadores da qualidade do solo (Quadro 1).

QUADRO 1 - Avaliação da qualidade biológica do solo após a implantação do cacau inoculado no PA Palmares I - Parauapebas-PA. Média de 10 repetições.

\begin{tabular}{|c|c|}
\hline Tratamento & Organismos Encontrados \\
\hline \multirow{2}{*}{ Testemunhas } & Aracnídeos \\
\cline { 2 - 2 } & Nematóides \\
\cline { 2 - 2 } & Collembola \\
\hline \multirow{2}{*}{ Inoculado } & Nematóides \\
\hline \multirow{2}{*}{} & Acarina \\
\cline { 2 - 2 } & Fungos micorrízicos \\
\cline { 2 - 2 } & Ascosporos \\
\cline { 2 - 2 } & Collembola \\
\hline
\end{tabular}


Conforme o quadro 1, constatou-se maior número de espécies da fauna edáfica, indicadores da qualidade biológica, na área de cacau inoculada com fungos micorrízicos arbusculares, quando comparada a área não inoculada. Sendo encontrado na área sem inoculação os seguintes organismos: aranhas, nematóides e colêmbolos e na área inoculada: nematóides, ácaros, fungos micorrízicos, ascosporos e colêmbolos. Os quais não foram contabilizados, visto que o objetivo deste não constitui-se em quantificar os organismos, mas sim determinar os tipos de organismo da macro e meso fauna encontrados na área de estudo. Observou-se também que não houve contaminação micorrízica na área que não foi inoculada no período analisado.

A presença dos organismos encontrados na área de monocultivo de cacau é devida ao fato desta área apresentar grande quantidade de matéria orgânica, consequentemente, observa-se maior presença destes organismos vivos no solo em especial os ácaros e os collembolos, pois a matéria orgânica ajuda a compor os corpos da maioria dos organismos edáficos, fornecendo elevadas quantidades de energia através dos resíduos vegetais e excreções das raízes (BRADY; WEIL, 2013, BERUDE et al., 2015; PRIMAVESI; PRIMAVESI, 2018).

Os dados encontrados corroboram com Geremia et al. (2015), Pompeu et al. (2016), Casaril et al. (2019) que observaram acréscimos e/ou respostas positivas da presença de fauna edáfica em áreas manejadas sob maior presença de matéria orgânica. Salientando-se que além do fato da matéria orgânica ser fonte de energia para os microrganismos, serve como fonte de nutrientes para as plantas (PRIMAVESI; PRIMAVESI, 2018).

A presença de maiores proporções dos ácaros está relacionada ao hábito alimentar destes organismos, que em sua grande maioria alimentam-se de matéria orgânica ou de microrganismos que se desenvolvem sobre este material em decomposição (SILVA et al., 2004; BEITIA et al., 2015). Os colêmbolos exercem um papel semelhante aos ácaros. Berude et al. (2015), Silva et al. (2020) pontuam a função detritívora dos colêmbolos, contribuindo para decomposição da matéria orgânica e controle das populações de microrganismos, em especial os fungos.

Ressalta-se também que a maior ocorrência de organismos na área inoculada, pode ser atribuída a extensão dos micélios externos dos fungos micorrízicos na rizosfera dos cacaueiros, que podem servir de alimento para organismos como colêmbolos e nematodóides (PAULA, (1992); BIANCIOTTO et al., (2012) citado por REIS et al., (2010). Silva et al. (2016) observaram maior comprimento dos micélios em áreas sob vegetações florestais e agrícolas. Assim, a extensão dos micélios pode ter aumentado a disponibilidade de alimentos para esses organismos.

A área de monocultivo de cacau inoculado com fungos micorrízicos arbusculares apresentou uma variabilidade maior de organismos presentes em uma única área, mostrando assim a viabilidade dos fungos micorrízicos na melhoria da qualidade do solo.

Pelo fato das micorrizas serem associações simbióticas e mutualistas, contribuem para a melhor fixação de fósforo e consequentemente diminuição do $\mathrm{pH}$, tornando este solo mais propício para o aparecimento de outros organismos indicadores da qualidade do solo, como os collêmbolos que apresentam papel fundamental na incorporação de restos vegetais no solo, aumentando assim, a disponibilidade de matéria orgânica e outros nutrientes (PRIMAVESI; PRIMAVESI, 2018) . 
Desta forma, a identificação destes organismos é de fundamental importância para obter informações acerca dos organismos existentes no solo e as interações que estes estabelecem com o meio, de modo a intervir de forma sustentável, obedecendo aos limites e potencialidades de cada agroecossistema.

\section{Parâmetros de Crescimento do Cacau}

Aos 19 meses após o plantio, as mudas de cacau inoculadas com fungos micorrízicos arbusculares se sobressaíram em comparação às mudas de cacau não inoculadas (testemunhas), pois no primeiro caso houve um aumento em todos os parâmetros observados (tabela 1).

TABELA 1. Avaliação da taxa de sobrevivência e parâmetros de crescimento das plantas de cacau no campo 19 meses após o plantio. Média de 422 plantas. PA Palmares I. Parauapebas-PA.

\begin{tabular}{lccccc}
\hline Tratamento & $\begin{array}{c}\text { Número } \\
\text { de } \\
\text { plantas } \\
\text { vivas }\end{array}$ & $\begin{array}{c}\text { \% de } \\
\text { plantas } \\
\text { vivas }\end{array}$ & $\begin{array}{c}\text { Altura das } \\
\text { plantas }(\mathbf{m})\end{array}$ & $\begin{array}{c}\text { Diâmetro do } \\
\text { colo }(\mathbf{m m})\end{array}$ & Número de folhas \\
$\begin{array}{l}\text { Testemunha } \\
\text { (não }\end{array}$ & $54 \mathrm{~b}$ & 12,79 & $1,06 \mathrm{~b}$ & $14,48 \mathrm{~b}$ & $10 \mathrm{~b}$ \\
$\begin{array}{l}\text { inoculado) } \\
\text { Inoculado }\end{array}$ & $330 \mathrm{a}$ & 78,2 & $1,90 \mathrm{a}$ & $48,48 \mathrm{a}$ & $28 \mathrm{a}$ \\
CV (\%) & 32,7 & - & 27,8 & 29,7 & 32,9 \\
\hline
\end{tabular}

$\mathrm{CV}=$ coeficiente de variação.

Médias seguidas pela mesma letra não diferem em teste Turkey de $5 \%$ de probabilidade.

Na tabela 1 observa-se que houve diferença significativa em todas as variáveis analisadas, sendo constatado o efeito benéfico dos FMA sobre o desenvolvimento de plantas de cacau aos 19 meses a campo. Posto que, ao estabelecer relação de comparação entre as plantas inoculadas com FMA e não inoculadas (testemunha) a primeira apresentou resposta superior em relação a segunda de: i) $64,41 \%$ quanto a porcentagem de plantas vivas; ii) $84 \mathrm{~cm}$ superior na média de altura de plantas; iii) incremento de $34 \mathrm{~mm}$ quanto a média de diâmetro do colo; iv) média de 18 folhas a mais quanto a variável número de folhas. Indicando que a adição de inóculos de Glomus. clarum proporcionaram um melhor desenvolvimento das plantas a campo.

Dentre os grupos de vegetais, as fruteiras são conhecidas por serem beneficiadas pela associação com as micorrízicas arbusculares, todavia existem diferenças no grau de micotrofismo que é refletido geralmente no crescimento vegetal (SILVA et al., 2009).

Os dados referentes à altura das plantas corroboram com os escritos por Machineski et al., (2009) que verificaram que plantas inoculadas com as espécies de FMA (G. margarita e G. clarum), apresentaram crescimento superior em altura. Soares e Martins (2000) verificaram que diferentes espécies de inóculos de FMAs utilizados em mudas de maracujazeiro amarelo proporcionaram aumentos significativos no crescimento e nos teores de nutrientes da parte aérea desta frutífera, quando comparados aos dos tratamentos sem inoculação.

Moreira e Siqueira (2002) afirmam que existe relação de proporcionalidade 
entre o tempo de exposição dos fungos às raízes hospedeiras, onde quanto maior o tempo de exposição, maiores são os benefícios para as plantas. Além disso,Berude et al., (2015) ao realizarem análise bibliográfica sobre as características da associação micorrízica observaram que a importância das hifas extracelulares, que ao se desenvolverem nas rizosferas contribuem para aspectos nutricionais das plantas e, consequentemente, aumentam a capacidade inicial de crescimento e estabilização

O bom desempenho desta frutífera também está relacionado a espécie que foi utilizada como inoculante, pois Vitorazzi Filho et al., (2017), Gomes et al., (2018) e Pinheiro et al., (2019) observaram resultados positivos no desenvolvimento de espécies frutíferas inoculadas com a espécies Glomus clarum.

Ao inocular mudas de porta-enxertos de citrus com fungos Glomus clarum e Glomus etunicatum Miranda et al., (2018) observaram que a presença dos fungos contribuiu para absorção de macronutrientes primários e secundários, sobretudo $\mathrm{N}, \mathrm{P}, \mathrm{K}$ e Mg.

Devido o preparo da área ter sido realizado pela prática do corte e queima, que resulta na diminuição dos microrganismos do solo (macro e meso fauna) e consequente perda da diversidade microbiana, pela redução de fonte de alimentos (REDIN et al., 2011) a maior ocorrência de táxons da fauna edáfica na área micorrizada pode ter favorecido o processo de decomposição de resíduos orgânicos e mineralização da matéria orgânica e formação de bioporos, predispondo condições favoráveis de crescimento vegetativo as mudas de cacau.

Lattuda et al. (2019) também verificaram que os fungos micorrízicos arbusculares otimizam o processo de adaptação de mudas de frutíferas nativas da família das Myrtaceae a campo, exercendo papel importante da associação em relação ao desenvolvimento vegetativo das espécies e acelerando o processo de adaptação a campo. Além disso, o fato da disponibilidade de $\mathrm{N}$ e $\mathrm{P}$ ser o principal fator limitante para o crescimento e a produtividade das plantas, torna os FMAS um insumo biológico com grande potencial para a agricultura (SIQUEIRA et al., 2002), comprovando assim a eficiência destes fungos na inoculação de frutíferas nas regiões tropicais, principalmente quando as plantas estão a campo em situações adversas de clima, fertilidade do solo e disponibilidade de água o que explica a taxa de $78,2 \%$ de sobrevivência das mudas, que no período analisado foi favorecido pela inoculação micorrízica com Glomus clarum.

\section{CONCLUSÃO}

A introdução de insumos biológicos (FMAs) no plantio de cacau contribuiu para a qualidade biológica do solo. Além disso, a associação de fungos micorrízicos arbusculares- Glomus clarum com mudas de cacau tornou possível a sua adaptação em condições de estresse, influenciando beneficamente na taxa de sobrevivência e em todos os parâmetros de crescimento avaliados. Desta forma, observa-se que a inoculação com FMAs é tecnologia promissora para adaptação de espécies frutíferas à campo . Sendo necessário a continuidade da investigação do uso do insumo biológico em cultivares de cacau, como forma de fomentar a lacuna de informações sobre o assunto na mesorregião sudeste do Pará. 


\section{AGRADECIMENTOS}

Os autores agradecem as bolsas de iniciação científica concedidas pelo CNPq - PIBIC - UFPA e CNPq - PROJETO REPENSA.

À Fapespa pela concessão de bolsa de estudo às mestrandas.

\section{REFERÊNCIAS}

BALOTA, E. L.: Manejo e qualidade biológica do solo. Edição revisada, Londrina: Midiograf, 280 p. 2018.

BEITIA,E. P. M.; PRAGER, M. S. de.; MONRROY, A. S.; PARADA, M. R. P. Populations of Mites, Collembola and other Mesofauna in an Inceptisol under Different Management. Revista Facultad Nacional de Agronomía Medellín. Medellín, v. 68, n. 1, p. 7411-7422, $2015 . \quad$ Disponível em: http://www.scielo.org.co/scielo.php?script=sci_arttext\&pid=S0304-

28472015000100003\&lng=en\&nrm=iso. Acesso em: 07 ago. 2020. http://dx.doi.org/10.15446/rfnam.v68n1.47828.

BERUDE, M. C.; GALOTE, J. K. B.; PINTO,P. H.; AMARAL, A. A. do. A mesofauna do solo e sua importância como bioindicador. Enciclopédia Biosfera, Goiânia, v.11 n.22, p.14-28. Disponível em: https://www.conhecer.org.br/enciclop/2015E/A\%20MESOFAUNA.pdf Acesso em: 07 ago. 2020.

BERUDE, M. C.; ALMEIDA, D. S.; RIVA, M. M.; CABANÊZ, P. A.; AMARAL, A. A. Micorrizas e sua importância agroecológica. Enciclopédia Biosfera, Goiânia, v.11 n.22; p. 132-146. Disponpivel em: https://www.conhecer.org.br/enciclop/2015E/Micorrizas.pdf Acesso em: 09 ago. 2020.

BERUDE, M. C.; ALMEIDA, D. S.; RIVA, M. M.; CABANÊZ, P. A.; AMARAL, A. A. Micorrizas e sua importância agroecológica. Enciclopédia Biosfera, Goiânia, v.11 n.22; p. 132-146. Disponpivel em: https://www.conhecer.org.br/enciclop/2015E/Micorrizas.pdf Acesso em: 09 ago. 2020.

BRADY, N. C ; WEIL, R. R. Elementos da natureza e propriedades dos solos. Porto Alegre: Bookman, 2013. 685 p. ISBN: 9788565837743.

CASARIL, E. C.; OLIVEIRA FILHO, L. C. I.; SANTOS, J. C. P.; ROSA, M. G. (2019). Fauna edáfica em sistemas de produção de banana no Sul de Santa Catarina. Revista Brasileira de Ciências Agrárias, v.14, n.1, p.1-12. 2019. Disponível em: http://www.agraria.pro.br/ojs2.4.6/index.php?journal=agraria\&page=article\&op=view\&pat h\%5B\%5D=agraria_v14i1a5613 Acesso em: 09 ago. 2020.

FARIAS, D. H.; PINTO, M. A. B.; CARRA, B.; SCHUCH, M. W.; SOUZA, P. V. D. Desenvolvimento de mudas de mirtileiro inoculadas com fungos micorrízicos arbusculares. Revista Brasileira de Fruticultura. Jaboticabal , v. 36, n. 3, p. 655-663. 2014.

FRANÇA, A. C.; CARVALHO, F. P.; FRANCO, M. H. R.; AVELAR, M.; SOUZA, B. P.; 
STUMER, S. L. Crescimento de mudas de cafeeiro inoculadas com fungos micorrízicos arbusculares. Agrária - Revista Brasileira de Ciências Agrárias. v.9, n.4, p.506-511, 2014.

Disponível

em:

http://sbicafe.ufv.br/bitstream/handle/123456789/11138/Rev.\%20Bras.\%20Ci\%c3\%aanc. \%20Agr\%c3\%a1rias_v.\%209_n.\%204_p.\%20506\%20-

\%20511_\%202014.pdf?sequence=1\&isAllowed=y Acesso em: 08 ago. 2020. DOI:10.5039/agraria.v9i4a3938.

GERDEMANN, J.W.; NICOLSON, T.H. Spores of mycorrhizal Endogone species extracted from soil by wt-sieving and decanting. Transactions of Britisch Mycological Society. v. 46, p. 235-244, 1963.

GEREMIA, E. V.; SEGAT, K. C.; FACHINI, I. A.; FONSECA, E. de O.; BARRETA,D. Fauna edáfica em Pastagem perene sob diferentes fontes de nutrientes . Scientia Agraria, v. 16, n. 4, 2016. Disponível em: https://revistas.ufpr.br/agraria/article/view/47802. Acesso em: 09 ago. 2020. doi:http://dx.doi.org/10.5380/rsa.v16i4.47802.

GOMES JR G. A.; PEREIRA, R. A.; GROSS, E. Dependência micorrizica e produção de glomalina por fungos micorrizicos arbusculares inoculados em gravioleira adubada com composto orgânico da casca de cacau. Ciência Agrícola, Rio Largo, v. 16, n. 3, p. 21 25, 2018. DOI: https://doi.org/10.28998/rca.v16i3.6378.

GOMIDE,P. H.; SILVA, M. L. N.; SOARES, C. R. F. S.; CARDOSO, E. L. FERNANDA, C.; LEAL,P. L.; MARQUES, R. M.; STURMER, S. L.Fungos micorrízicos arbusculares em fitofisionomias do Pantanal da Nhecolândia, Mato Grosso do Sul. Revista Brasileira de Ciências do Solo. v.38 n.4 Viçosa, 2014. DOI: https://doi.org/10.1590/S010006832014000400007.

IBGE - Instituto Brasileiro de Geografia e Estatística - Levantamento Sistemático da Produção Agrícola - LSPA. Brasília - DF, 108p. Disponível em: $\mathrm{ftp}: / / \mathrm{ftp}$.ibge.gov.br/Producao_Agricola/Levantamento_Sistematico_da_Producao_Agrico la_[mensal]/Fasciculo/2017/Ispa_201712.pdf. Acesso em: 1 agost. 2020

JENKINS, W.R. A rapid centrifugal-floration technique for separating nematodes from soil. Plant Disease Report, v.48, 1964. 692p.

JESUS, A. R.; SALVI, E. J. N. R.; CHAGAS; COSTA, G. R.; SOUZA, L. G. Cultivo de cacau orgânico. Dossiê técnico. Serviço Brasileiro de Respostas Técnicas Instituto Euvaldo Lodi - IEL/BA, 2013.

LATTUADA, D.; RIETH, S.; BACK, M.; SOUZA, P. Interação entre endomicorrizas e frutíferas nativas (Myrtaceae) no Rio Grande do Sul. Ciência Florestal. v. 29, n. 4, p. 1726-1736, 2019. Disponível em: https://periodicos.ufsm.br/cienciaflorestal/article/view/37389 Acesso em: 09 ago. 2020 Doi: https://doi.org/10.5902/1980509837389

LEMOS, R. C. Manual de descrição e coleta de solo no campo. $3^{\underline{a}}$ Ed. Campinas, 
Sociedade Brasileira de Ciência do Solo, 2000.84 p.

LIMA, K. B.; MARTINS, M. A.; FREITAS, M. S. M.; OLIVARES, F. L. Fungos micorrízicos arbusculares, bactérias diazotróficas e adubação fosfatada em mudas de mamoeiro. Revista Brasileira de Fruticultura. Jaboticabal, v. 33, n. 3, p. 932940, $2011 . \quad$ Disponível em: http://www.scielo.br/scielo.php?script=sci_arttext\&pid=S010029452011000300029\&lng= en\&nrm=iso>. Acesso em: 08 ago. 2020 doi: http://dx.doi.org/10.1590/S010029452011000300029.

MACHINESKI, O.; SILVA, A. P. D.; TRUBER, P. V.; GARCIA, T. L.; BALOTA, E. L. Synergismuss cyentifica UTFPR, Pato Branco, v. 4, n. 1, p. 1-3, 2009. Disponível em: http://revistas.utfpr.edu.br/pb/index.php/SysScy/article/view/492 Acesso em: 08 ago. 2020.

MANTOVANELLI, B. C.; CAMPOS, M. C. C.; ALHO, L. C.; FRANCISCON, U.; NASCIMENTO, M. F.; SANTOS, L. A. C. Distribuição espacial dos componentes de acidez do solo em área de campo natural na região de Humaitá, Amazonas. Revista de Ciências Agroambientais, Rio Verde. v. 14, n. 1, p. 1-9, 2016.

MIRANDA, P. B.; HENTZ, A. M.; KUPPER, K. C. Mycorrhizal dependence of citrus rootstock. Revista Brasileira de Fruticultura. Jaboticabal, v. 40, n. 3, e-762, 2018. Disponível em: https://www.scielo.br/pdf/rbf/v40n3/0100-2945-rbf-40-3-e-762.pdf Acesso em : 25 ago. 2020.

MOREIRA, F. M. S.; SIQUEIRA, J. O. Microbiologia e bioquímica do solo. Lavras: Universidade Federal de Lavras, 2002. v.1.291 p.

NASCIMENTO, J. M. L.; MENEZES, Souza, K. M.; QUEIROZ, M. A. Á.; MELO, A. M. Y. Crescimento inicial e composição bromatológica de plantas de pornuncia adubadas com fósforo e inoculadas com fungos micorrízicos arbusculares. Revista Brasileira de Saúde e Produção Animal. Salvador, v.17, n.4, p.561-571. 2016. DOI: http://dx.doi.org/10.1590/S1519-99402016000400001.

OLIVEIRA, L. P. Programa de Desenvolvimento da Cadeia Produtiva do Cacau no Pará- PRÓCACAU- 2011/2019. Belém, Pará: SEDAP, 56 p. 2016. Disponível em: http://www.sedap.pa.gov.br/sites/default/files/Projeto\%20de\%20Cacau\%20\%20formatad 0\%20_\%20final.pdf. Acesso em: 01 ago. 2020.

PARÁ. GOVERNO DO PARÁ. Secretaria de Desenvolvimento da Pecuária e da Pesca. Estratégias para o desenvolvimento sustentável: produção e verticalização do cacau. Programa Pará 2030. SEDAP. Belém-PA, 2016. (para2030.com.br). Disponível em: http://para2030.com.br/wp-content/uploads/2017/08/Cacau.pdf. Acesso em: 01 ago. 2020.

PEREIRA, F. D.; MELLO, A. H de; CORREA, H. de S.; MICHELOTTI, F.; MANESCHY, R. Q.; KNOECHELMANN, C. M. Distribuição de fungos micorrízicos nos assentamentos 
Palmares e Araras. Revista Agroecossistemas, v. 2, n. 1, p. 2-7, 2013. ISSN 23180188.

Disponível

em:

https://periodicos.ufpa.br/index.php/agroecossistemas/article/view/1173. Acesso em: 08 ago. 2020. doi:http://dx.doi.org/10.18542/ragros.v2i1.1173.

PIMENTEL GOMES, F. Curso de Estatística Experimental. Piracicaba: Ed. Livraria Nobel. S.A. Editora - Distribuidora, 1987.

PINHEIRO,E. M.; NOBRE, C. P.; COSTA,T. V.; TAVARES, O. C. H.; ARAUJO, J. R. Arbuscular mycorrhizal fungi in seedling formation of barbados cherry (Malpighia emarginata D.C.). Revista Caatinga, Mossoró , v. 32, n. 2, p. 370-380, 2019. Disponível em: http://www.scielo.br/scielo.php?script=sci_arttext\&pid=S198321252019000200370\&Ing=en\&nrm=iso. Acesso em: 09 ago. 2020 doi: https://doi.org/10.1590/1983-21252019v32n210rc.

POMPEO, P. N.; OLIVEIRA FILHO, L. C. I.; KLAUBERG FILHO, O.; MAFRA, A.L.; BARETTA, C.R.D.M.; BARETTA, D. Diversidade de Coleoptera (Arthropoda: Insecta) e atributos edáficos em sistemas de uso do solo no Planalto Catarinense. Scientia Agraria, v.17, n.1, p.16-28, 2016.2 Disponível em: https://revistas.ufpr.br/agraria/article/view/46535/29371 Acesso em: 09 ago. 2020 https://doi.org/10.5380/rsa.v17i1.46726.

PRIMAVESI, A.; PRIMAVESI, A. A biocenose do solo na produção vegetal e deficiências minerais em culturas, nutrição e produção vegetal. São Paulo: Expressão Popular. 608 p. 2018.

REDIN, M.; SANTOS, G. F.; MIGUEL, P.; DENEGA, G. L.; LUPATINI, M.; DONEDA, A.; SOUZA, E. L. Impactos da queima sobre atributos químicos, físicos e biológicos do solo. Ciência Florestal, v. 21, p. 381-392, 2011. Disponível em: https://periodicos.ufpa.br/index.php/agroecossistemas/article/view/4781/4396 Acesso em 20 abr. 2020.

REIS, V. M.; ANDRADE, G.; FARIA, S. M.; SILVEIRA, A. P. D. Interações de fungos micorrízicos arbusculares com outros microrganismos do solo. In: SIQUEIRA et al. (Eds) Micorrizas: 30 anos de pesquisas no Brasil. Lavras: UFLA, 2010. 716 p.

RODRIGUES, L. A.; BARROSO, D. G.; FIQUEIREDO, A. M. M. A.; Fungos micorrízicos arbusculares no crescimento e na nutrição Mineral de mudas de Tectona grandis $L$. F. Ciência Florestal, Santa Maria, v. 28, n. 1, p. 25-34, 2018. DOI: http://dx.doi.org/10.5902/1980509831572.

SANTOS, D. R.; SILVA, M. M. Agrobiodiversidade em áreas cultivadas com cacau em Altamira-Pará, Amazônia Oriental. Revista Brasileira de Agroecologia. [S.I] v. 12, n. 3, sep. $2017 . \quad$ Disponível em: http://revistas.abaagroecologia.org.br/index.php/rbagroecologia/article/view/21962. Acesso em: 25 ago. 2020.

SCHEMBERGUE, A.; CUNHA, D. A.; CARLOS, S. M.; PIRES, M. V.; FARIA, R. M. Sistemas agroflorestais como estratégia de adaptação aos desafios das mudanças 
climáticas no Brasil. Revista de Economia e Sociologia Rural, Brasília, v. 55, n. 1, p. 9-28, mar. 2017. Disponível em: http://www.scielo.br/pdf/resr/v55n1/1806-9479-resr-5501-00009.pdf. 20 julh. 2020.

SILVA, C. F.; PEREIRA, M. G.; SANTOS, V. L.; MIGUEL, D. L.; SILVA, E. M. R. Fungos micorrízicos arbusculares: composição, comprimento de micélio extrarradicular e glomalina em áreas de Mata Atlântica. Rio de Janeiro. Ciência Florestal. Santa Maria, v. 26, n. 2, p. 419-430, 2016. Disponível em: http://www.scielo.br/scielo.php?script=sci_arttext\&pid=S198050982016000200419\&lng= en\&nrm=iso Acesso em: 08 ago. 2020.

SILVA, E. S.; MORAES, G. J. de; KRANTZ, G. W. Diversity of edaphic Rhodacaroid mites (Acari: Mesostigmata: Rhodacaroidea) in natural ecosystems in the state of São Paulo, Brazil. Neotropical Entomology. Londrina, v. 33, n. 5, p. 547-555. 2004. Disponível em: http://www.scielo.br/scielo.php?script=sci_arttext\&pid=S1519566X2004000500002\&lng=en\&nrm=iso. Acesso em: 08 ago. 2020. http://dx.doi.org/10.1590/S1519-566X2004000500002.

SILVA, J.; KUBIAK, K.; ZARZICKI, L.; DONAZZOLO, J.; TESSARO, D. Diversidade morfológica de colêmbolos em sistemas agroflorestais no Sudoeste do Paraná. Research, Society and Development. Research, Society and Development, v. 9, n. 6. 2020. DOI: http://dx.doi.org/10.33448/rsd-v9i6.3606.

SILVA, T. F. B.; SANTOS, A. B. S.; ROZAS, C. E. O.; PAIVA, L. M. Influência da densidade de fungos micorrízicos arbusculares na produção de maracujazeiro-doce (passiflora alata curtis). Revista Caatinga, Mossoró, v.22, n.4, p.1-6, 2009. Disponível em: https://periodicos.ufersa.edu.br/index.php/caatinga/article/view/364. Acesso em: 08 ago. 2020.

SIQUEIRA, J. O; LAMBAIS, M. R; STÜRMER, S. L. Fungos Micorrízicos Arbusculares: Características, associação simbiótica e aplicação na agricultura. Biotecnologia Ciência \& Desenvolvimento, Brasília, v. 4, n. 25, 2002. Disponível em: www.biotecnologia.com.br/revista/bio25/fungos.pdf. Acesso em: 21 ago. 2013.

SOARES, A. C. F.; SOUSA, C. S.; GARRIDO, M. S.; LIMA, F. S. Fungos micorrízicos arbusculares no crescimento e nutrição de mudas de jenipapeiro. Revista Ciência Agronômica, v. 43, n. 1, p. 47-54, 2012.Disponível em: https://www.scielo.br/scielo.php?script=sci_arttext\&pid=S1806-66902012000100006 Acesso em: 08 ago. 2020 http://dx.doi.org/10.1590/S1806-66902012000100006.

SOARES, A. C. F; MARTINS, M. A. Influência de fungos micorrízicos arbusculares, associada à adição de compostos fenólicos, no crescimento de mudas de maracujazeiro amarelo (passiflora edulis f. Flavicarpus). Revista Brasileira de Ciência do Solo, [S. I.], v. 24, p 731-740, 2000. Disponível em: https://www.scielo.br/scielo.php?script=sci_abstract\&pid=S010006832000000400005\&ln $\mathrm{g}=\mathrm{en} \& \mathrm{nrm}=$ iso\&tIng=pt Acesso em: 08 ago. 2020.

VITORAZI FILHO, J. A.; FREITAS, M. S. M.; MARTINS,M .A.; SANTOS, P. C.; 
CARVALHO,A.J.C. Fungos micorrizicos arbusculares e adubação fosfatada em mudas de caramboleira. Agrária - Revista Brasileira de Ciências Agrárias, v.12, n.1, p.14-19, 2017. Disponível em: http://www.agraria.pro.br/ojs2.4.6/index.php?journal=agraria\&page $=$ article $\& o p=v i e w \& p a t h \% 5 B \% 5 \mathrm{D}=$ agraria_v12i1a 5410\&path\%5B\%5D=4945 Acesso em: 09 ago. 2020. doi:10.5039/agraria.v12i1a5410. 